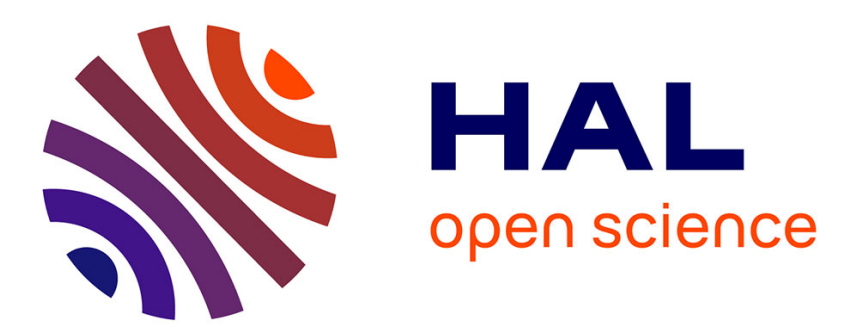

\title{
Optimal Design of a PMU-Based Monitoring Architecture for Power Systems
}

Didier Georges

\section{To cite this version:}

Didier Georges. Optimal Design of a PMU-Based Monitoring Architecture for Power Systems. 8th IFAC Symposium on Power Plant and Power System Control (PP\&PSC 2012), Sep 2012, Toulouse, France. hal-00834353

\section{HAL Id: hal-00834353 https://hal.science/hal-00834353}

Submitted on 14 Jun 2013

HAL is a multi-disciplinary open access archive for the deposit and dissemination of scientific research documents, whether they are published or not. The documents may come from teaching and research institutions in France or abroad, or from public or private research centers.
L'archive ouverte pluridisciplinaire HAL, est destinée au dépôt et à la diffusion de documents scientifiques de niveau recherche, publiés ou non, émanant des établissements d'enseignement et de recherche français ou étrangers, des laboratoires publics ou privés. 


\title{
Optimal Design of a PMU-Based Monitoring Architecture for Power Systems
}

\author{
Didier Georges* \\ * Grenoble INP / Univ Grenoble 1 / Univ Grenoble 3 / CNRS, \\ GIPSA-lab, F-38402 Saint Martin d'Hères, \\ (e-mail: didier.georges@gipsa-lab.grenoble-inp.fr).
}

\begin{abstract}
In this paper, a methodology for designing an optimal monitoring architecture by using a limited number of PMU (Phasor Measurement Units) and a PDC (Phase Data Concentrator) is proposed. The optimal design problem consists in defining the optimal location of both the PMU and the PDC by maximizing the expected value of the trace of the observability gramian of the power system over a large number of set point scenarii, while minimizing the communication infrastructure cost. Furthermore, a nonlinear dynamical EKF (Extended Kalman Filtering) state-observer is proposed. This state-observer allows to take transient phenomena into account for wide-area power systems described by algebraicdifferential equations, without needing nonlinear inversion techniques. Application to a 5-bus power system is also presented and demontrates the effectiveness of the approach.
\end{abstract}

Keywords: Power system monitoring, optimal design of monitoring achitecture, sensor networks, observability gramian, optimal location of PMU and PDC, dynamical state estimation, extended Kalman filtering.

\section{NOMENCLATURE}

$\begin{array}{cc}\text { PMU } & \text { Phasor Measurement Unit. } \\ \text { PDC } & \text { Phasor Data Concentrator. } \\ \text { EKF } & \text { Extended Kalman Filtering. } \\ & \text { Subscript } i \text { denotes the ith generator. } \\ \delta_{i} & \text { Angle of the ith generator in radian. } \\ \theta_{i} & \text { Bus } i \text { phase angle, in radian. } \\ \omega_{i} & \text { Relative speed in rad/s. } \\ P_{i}^{m} & \text { Mechanical input power, in p.u.. } \\ P_{G i} & \text { Active power delivered, in p.u.. } \\ Q_{G i} & \text { Reactive power, in p.u.. } \\ E_{q i}^{\prime} & \text { Transient EMF in quadrature axis, in p.u.. } \\ \omega_{0} & \text { Synchronous machine spedd, in rad/s. } \\ D_{i} & \text { Per unit damping constant. } \\ H_{i} & \text { Inertia constant in second. } \\ T_{d 0 i}^{\prime} & \text { Direct axis transient short circuit time constant, in s. } \\ x_{d i} & \text { Direct axis reactance, in p.u.. } \\ x_{d i}^{\prime} & \text { Direct axis transient reactance, in p.u.. } \\ K_{a i} & \text { Exciter gain, in p.u.. } \\ T_{a i} & \text { Exciter time constant in p.u.. } \\ E_{f d i} & \text { Exciter voltage in p.u.. } \\ B_{i j} & \text { Susceptance of admittance matrix element } i, j . \\ G_{i j} & \text { Conductance of admittance matrix element } i, j . \\ P_{L i} & \text { Load active power at bus } i, \text { in p.u.. } \\ Q_{L i} & \text { Load reactive power at bus } i, \text { in p.u.. } \\ V_{i} & \text { Voltage at bus } i, \text { in p.u.. } \\ C_{i} & \text { Set of bus indices connected to bus } i, \text { including } i .\end{array}$

\section{INTRODUCTION}

A big challenge for modern monitoring architectures of wide-area power systems is to provide some reliable information on the health of the system, especially in the context of large transients. Nowadays communication networks plays a key role for providing reliable measurement data with minimum latency in the context of wide-area power systems. Wireless communications (both WIMAX and $3 \mathrm{G} / 4 \mathrm{G}$ ) are expected to wide-area data network coverage (see Akyol (2010)), especially in remote regions where conventional public communication networks are not available. Traditional monitoring of power grids is ensured by Remote Terminal Units (RTU), which provide real/reactive power flows, real/reactive power injections and voltage magnitude measurements. The introduction of PMU by Phadke (1983) offers additional measurements such as voltage and current phasor measurements. PMU can provide very accurate data since they are synchronized from the common global positioning system (GPS) radio clock. However the use of PMU is more demanding in terms of data flow rates than traditional RTU (see Narendra (2008)).

Previous works have been dedicated to the location of the optimal location of PMU (see Baldwin (1993), Gou (2008), $\mathrm{Xu}(2004)$.)

In this paper, we will consider several issues:

- First, in order to effectively track the transient behavior of power systems, we will consider dynamical observability rather than the conventional static observability.

- Second, the system is supposed to be already equiped with convention RTU, since in practice, due to various resource limitations, the practical problem is to determine the location of PMU, which are added incrementally. 
- Third, one main issue is to provide engineers with monitoring infrastructure design tools. The infrastructure must be understood as the choice of the power system state variables to be measured (optimal location of a fixed number of PMU), together with the communication network which will be used for transmitting sensor measurements to a data center.

To the best of my knowledge, the here-proposed codesign approach was not studied in previous works. This paper extends the approach proposed in Nguyen (2008) by including communication network design. Furthermore, Extended Kalman Filtering is used to design a dynamical state observer for wide-area power systems described by algebraic-differential equations, without needing nonlinear inversion techniques.

The paper is now organized as follows: Section 2 is dedicated to modelling of wide-area power systems. In section 4, an optimal design methodology for wide-area monitoring is proposed based on observability gramian recalled in section 3. Section 5 describes an EKF-based dynamical state-observer. Section 6 is devoted to application of the methodology to a 5-bus power system. Finally some conclusions and perspectives are provided.

\section{POWER SYSTEM MODELING FOR WIDE-AREA MONITORING}

We consider a multi-machine power system, with $N$ generators connected to a grid made of $M$ buses, where $M \geq N$. Using a one-axis model of each generator ${ }^{1}$, the model of the ith generator equiped with a dynamical exciter can be expressed as follows (see Ilic (2000) for example):

\section{Mechanical Dynamics of Generator $i$ :}

$$
\begin{aligned}
\dot{\delta}_{i} & =\omega_{i}, \\
\dot{\omega}_{i} & =-\frac{D_{i}}{2 H_{i}} \omega_{i}+\frac{\omega_{0}}{2 H_{i}}\left(P_{i}^{m}-P_{G i}\right) .
\end{aligned}
$$

\section{Electrical Dynamics of Generator $i$ :}

$$
\begin{aligned}
\dot{E}_{q i}^{\prime} & =\frac{1}{T_{d 0 i}^{\prime}}\left(E_{f d i}-\frac{x_{d i}}{x_{d i}^{\prime}} E_{q i}^{\prime}-\frac{\left(x_{d i}-x_{d i}^{\prime}\right)}{x_{d i}^{\prime}} V_{i} \cos \left(\delta_{i}-\theta_{i}\right),\right. \\
\dot{E}_{f d i} & =-\frac{1}{T_{a i}}\left(E_{f d i}-E_{f d 0 i}\right)+\frac{K_{a i}}{T_{a i}}\left(V_{\text {refi }}-V_{i}\right) .
\end{aligned}
$$

Electrical Equations at Generator bus $i, i=1, \ldots, N$ :

$$
\begin{aligned}
P_{G i} & =\frac{E_{q i}^{\prime} V_{i}}{x_{d i}^{\prime}} \sin \left(\delta_{i}-\theta_{i}\right), \\
Q_{G i} & =\left(E_{q i}^{\prime} V_{i} \cos \left(\delta_{i}-\theta_{i}\right)-V_{i}^{2}\right) / x_{d i}^{\prime}, \\
P_{G i} & =\sum_{k \in C_{i}} V_{i} V_{k}\left(G_{i k} \sin \theta_{i k}-B_{i k} \cos \theta_{i k}\right)+P_{L i}, \\
Q_{G i} & =\sum_{k \in C_{i}} V_{i} V_{k}\left(G_{i k} \cos \theta_{i k}+B_{i k} \sin \theta_{i k}\right)+Q_{L i},
\end{aligned}
$$

where $\theta_{i k}=\theta_{i}-\theta_{k}$.

1 For sake of simplicity and without any restriction, we do not consider a two-axis or a more detailed model of the generator.
Electrical Equations at the Non Generator Bus $j$, $j=1, \ldots, M-N$ :

$$
\begin{aligned}
P_{L j}+\sum_{k \in C_{j}} V_{j} V_{k}\left(G_{j k} \sin \theta_{j k}-B_{j k} \cos \theta_{i k}\right) & =0, \\
Q_{L j}+\sum_{k \in C_{j}} V_{j} V_{k}\left(G_{j k} \cos \theta_{j k}+B_{j k} \sin \theta_{j k}\right) & =0 .
\end{aligned}
$$

The overall dynamical model of the power system may be expressed as the following algebraic-differential system:

$$
\begin{aligned}
& \dot{x}=F(x, z, u), \\
& 0=G(x, z, w),
\end{aligned}
$$

where $x$ denotes the vector of the $N$ generator state variables $\left(\delta_{i}, \omega_{i}, E_{q i}^{\prime}, E_{f d i}\right), i=1, \ldots, N$ and possibly the state variables of additional controllers such as PSS or FACTS, $z$ is the $2 M$ vector of the voltage magnitude and phase angle at the $M$ buses. $u$ is the vector of reference control inputs, i.e. the mechanical power and the reference inputs of each controllers. $w$ is the vector of load currents at each bus. $w$ can also include interconnection variables if the studied system is part of a larger power system. $F$ denotes the vector field of the differential part of the statespace representation, while $G$ is a nonlinear function with the same dimensions as the ones of vector $z$.

For the goal of both simulation and state-observer design, we can find it convenient to use a differential version of the algebraic equations by introducing the time-derivative of $G$ :

$$
\dot{G}(x, z, w)=\frac{\partial G}{\partial x} \dot{x}+\frac{\partial G}{\partial z} \dot{z}+\frac{\partial G}{\partial w} \dot{w}
$$

and the condition $\dot{G}=0$. Rather than using this condition, it is more appropriate to reinforce numerical stability by introducing a stabilizing term as follows:

$$
\dot{G}(x, z, w)=-\frac{1}{\epsilon} G(x, z, w),
$$

with $\epsilon>0$. This equation is equivalent to

$$
\dot{z}=-\frac{\partial G^{-1}}{\partial z}\left[\frac{\partial G}{\partial x} F(x, z, u)+\frac{\partial G}{\partial w} \dot{w}+\frac{1}{\epsilon} G(x, z, w)\right]
$$

provided that the Jacobian matrix $\frac{\partial G}{\partial z}$ has full rank.

\section{Some remarks:}

(1) The classical approach consists in using the implicit function theorem, in order to locally express $z$ as a function $\Psi(x, w)$ provided that the Jacobian matrix $\frac{\partial G}{\partial z}$ has full rank. In most of the cases, it is not possible to get an explicit expression of $\Psi(x, w)$ and some Newton-Raphson-like methods are thus needed to compute $z$. The here-proposed differential version avoids such iterative computations of the implicit solution, except for the initial state.

(2) Equation (14) may be interpreted as a singularlyperturbated version of $G(x, z, w)=0$, since it may be rewritten as $\epsilon \dot{G}(x, z, w)=-G(x, z, w)$. 
(3) It can be easily shown that the solution of (14) is given by

$$
G(x(t), z(t), w(t))=e^{-\frac{t}{\epsilon}} G(x(0), z(0), w(0)) .
$$

This means that the trajectories of $x(t), z(t), w(t)$ are constrained to remain on the manifold defined by $G(x, z, w)=0$, when the initial states $x(0), z(0), w(0)$ are such that $G(x(0), z(0), w(0))=0$.

(4) It also means that any linearized dynamics around a given equilibrium state exhibits $2 M$ eigenvalues equal to $-\frac{1}{\epsilon}$ (in order to prove this assertion, consider the change of coordinates $z \rightarrow G(x, z, w))$.

In addition to the state-representation (11)-(12), we need to define the measurement vector $y$ :

$$
y=H(x, z)
$$

where $H(x, z)$ defines the measured variables as a function of the states $x$ and $z$. When some traditional sensors are used to measure active and reactive powers, the related measured variables will be expressed as a nonlinear function of the state. When some sensors (PMU for example) are used to measure phase angle and voltage magnitudes, the related component of $y$ will be simply one of the state components. Again, if some current measurements are performed, the related output will be a nonlinear function of the states.

Finally, a nonlinear fully-differential state representation of the power system is given by

$$
\begin{aligned}
\dot{x} & =F(x, z, u), \\
\dot{z} & =-\frac{\partial G^{-1}}{\partial z}\left[\frac{\partial G}{\partial x} F(x, z, u)+\frac{\partial G}{\partial w} \dot{w}\right. \\
& \left.+\frac{1}{\epsilon} G(x, z, w)\right], \\
y & =H(x, z) .
\end{aligned}
$$

In what follows, we will assume that the vector of load and interconnection currents $w$ are known via measurements, together with their time-derivatives (through some appropriate filters for example) ${ }^{2}$.

\section{OBSERVABILITY ANALYSIS BASED ON THE OBSERVABILITY GRAMIAN}

For asymptotically stable linear systems (i.e. the spectrum of matrix $A$ belong the left part of the complex plane) defined by

$$
\begin{aligned}
& \dot{x}=A x+B u, \\
& y=C x+D u,
\end{aligned}
$$

a measure of observability is given by the output energy function generated by any intial state $x_{0}$ (when $u=0$ ) and given by

$$
E_{o}\left(x_{0}\right)=\int_{0}^{\infty}\|y(t)\|^{2} d t=x_{0}\left[\int_{0}^{\infty} e^{A^{T} t} C^{T} C e^{A t} d t\right] x_{0} .
$$

2 An extension to the case of non measured $w$ is possible by introducing an extended version of (18)-(20) obtained by adding the dynamics $\dot{w}=0$.
The matrix $W_{o}=\int_{0}^{\infty} e^{A^{T} t} C^{T} C e^{A t} d t$ is called observability Gramian of the system and is given as solution of the following Lyapunov equation:

$$
A^{T} W_{o}+W_{o} A+C^{T} C=0 .
$$

When the pair $(C, A)$ is detectable ${ }^{3}, W_{o}$ is a nonnegative definite matrix.

In Zhou (1999), the notion of Gramian has been extended to the case of unstable systems, by considering the solutions $Y, W_{o}$ of the following algebraic matrix equations:

$$
\begin{aligned}
& A Y+Y A^{T}-Y C^{T} C Y=0, \\
& A_{o}^{T} W_{o}+W_{o} A_{o}+C^{T} C=0,
\end{aligned}
$$

where $A_{o}=A-Y C^{T} C$ and with $W_{o}=W_{o}^{T} \geq 0$ and $Y=Y^{T} \geq 0$.

The first (Bernoulli) equation ensures the projection of the unstable eigenvalues of $A$ to the left part of the complex plane, symmetrically with respect to the imaginary axis (by using an "implicit" Kalman filter). When the system is stable, we use in fact the trivial solution $Y=0$. As a consequence $W_{o}$ may be used as a measure of the "level of observability" induced by a particular choice or location of sensors in the system. An optimal location of sensors will consist for instance in maximizing the minimum singular value of $W_{o}$ (see Georges (1995)) or the trace of $W_{o}$, which represents the sum of the singular values of $W_{o}$ (see Georges (2011)). This is precisely the approach we will retain to propose a methodology for designing an optimal monitoring architecture. In order to do so, we will consider the local linearization of the system (18)-(19)-(20) around any equilibrium set point (denoted by the subscript "e"):

$$
\begin{aligned}
& \dot{\tilde{x}}=A_{e}^{1} \tilde{x}+A_{e}^{2} \tilde{z}+B_{e}^{1} \tilde{u}, \\
& \dot{\tilde{z}}=A_{e}^{3} \tilde{x}+A_{e}^{4} \tilde{z}+B_{e}^{2} \tilde{u}+B_{e}^{3} \tilde{w}, \\
& \tilde{y}=C_{e}^{1} \tilde{x}+C_{e}^{2} \tilde{z},
\end{aligned}
$$

where $A_{e}^{1}, A_{e}^{2}$ and $B_{e}^{1}$ are the Jacobian matrices of $F$ with respect to $x, z$ and $u$ respectively, while $A_{e}^{3}, A_{e}^{4}, B_{e}^{2}$ and $B_{e}^{3}$ are the Jacobian matrices of $z$ vector field with respect to $x, z, u$ and $w$ respectively, and finally, $C_{e}^{1}$ and $C_{e}^{2}$ are the Jacobian matrices of $H$ with respect to $x$ and $z$, evaluated at the equilibrium point. . denotes the discrepancy between the variable and its equilibrium value.

The observability Gramian of the power system is then computed as solution of (25) and (26), where

$$
A=\left(\begin{array}{cc}
A_{e}^{1} & A_{e}^{2} \\
A_{e}^{3} & A_{e}^{4}
\end{array}\right), C=\left(\begin{array}{ll}
C_{e}^{1} & C_{e}^{2}
\end{array}\right) .
$$

\section{DESIGN OF A MONITORING ARCHITECTURE BASED ON THE OPTIMAL LOCATION OF PMU AND PDC}

We are now interested in adding $N_{p}$ PMU to an existing monitoring infrastructure equiped with both traditional

3 A system is said to be detectable, if and only if the non observable states of the system are asymptotically stable. For each non observable state, there is a null eigenvalue in the observability Gramian matrix. 
P, Q, V sensors or already existing PMU. For sake of simplicity, we consider that the PMU are connected to a unique PDC, which is itself connected to a SCADA master station, according to fig. (1).

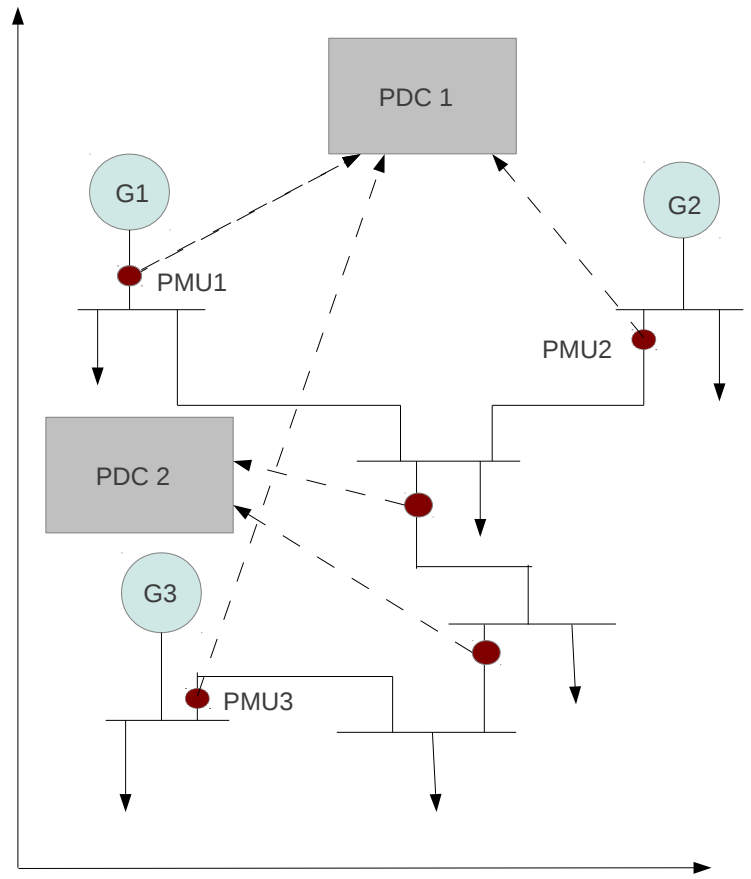

0xy

Fig. 1. Example of the location of 3 additional PMU connected to a PDC.

Our goal is now to derive an optimal monitoring architecture by taking three main objectives into account:

(1) The PMU must be located to adequate places in order to ensure the maximum observability of the power system, for a large number of set points.

(2) The PMU placement must be compatible with communication constraints and cost limitations (for example if some WIMAX, coaxial or optical communications are used, the distance between each PMU and the PDC is constrained to maintain minimal data flow rates and to avoid prohibitive infrastructure costs.)

(3) The optimal location of the PDC must be ensured to account the objectives of maximizing the observability while reducing the communication infrastructure induced costs and satisfying the communication constraints.

These objectives can be fulfilled by solving the following mixed integer programming problem:

$$
\begin{array}{rl}
\max _{S_{i j} \in\{0,1\}, x_{p d c}, y_{p d c}} & E\left[\operatorname{trace}\left(W_{o}(S, \omega)\right)\right] \\
& -C_{c}\left(S, x_{p d c}, y_{p d c}\right)
\end{array}
$$

subject to

$$
d(S) \leq \bar{d}
$$

$$
\begin{aligned}
& \sum_{j=1}^{N_{s}} S_{i j}=1, \\
& \sum_{i=1}^{N_{p}} S_{i j}=1,
\end{aligned}
$$

where $S_{i j}$ denotes the $i j$ th element of the PMU-selection matrix $S$ and $x_{p d c}, y_{p d c}$ is the spatial coordinates vector of the PDC location. $S_{i j}$ is equal to 1 , when the ith $\mathrm{PMU}$ is located in order to measure the jth partition (some current and voltage magnitudes, phase angle) of the state vector $\left(x^{T}, z^{T}\right)^{T}$, where $N_{s}$ is the number of state vector partitions. $E[$.$] is the expected value defined on a$ probability space defined by a large number $N_{s c}$ of scenarii $\omega_{i}$ corresponding each to one equilibrium set point of the power system. Each scenario has its own probability of occurence $P\left(\omega_{i}\right)$. Consequently,

$$
E\left[\operatorname{trace}\left(W_{o}(S, \omega)\right)\right]=\sum_{i=1}^{N_{s c}} \operatorname{trace}\left(W_{o}\left(S, \omega_{i}\right)\right) P\left(\omega_{i}\right) .
$$

Each $W_{o}\left(S, \omega_{i}\right)$ is solution of (25) and (26):

$$
\begin{array}{r}
A\left(\omega_{i}\right) Y\left(S, \omega_{i}\right)+Y\left(S, \omega_{i}\right) A^{T}\left(\omega_{i}\right) \\
-Y\left(S, \omega_{i}\right) C^{T}\left(S, \omega_{i}\right) C\left(S, \omega_{i}\right) Y\left(S, \omega_{i}\right)=0, \\
A_{o}^{T}\left(\omega_{i}\right) W_{o}\left(S, \omega_{i}\right)+W_{o}\left(S, \omega_{i}\right) A_{o}\left(\omega_{i}\right) \\
+C^{T}\left(S, \omega_{i}\right) C\left(S, \omega_{i}\right)=0,
\end{array}
$$

where $A_{o}=A-Y C^{T} C$.

$C_{c}\left(S, x_{p d c}, y_{p d c}\right)$ is the communication infrastructure cost induced by the location of both the $N_{p}$ PMU and the DPC. $C_{c}$ is defined as a function of the length of the $N_{p}$ links connecting each PMU to the PDC. The constraint (32) is introduced to limit the length of the $N_{p}$ links, in order to avoid prohibitive communication infrastructure costs and to maintain a minimum level of data flow rates. $d(S)$ is the distance vector defined by component

$$
d_{i}\left(S_{i}\right)=\sqrt{\left(x\left(\bar{S}_{i}\right)-x_{p d c}\right)^{2}+\left(y\left(\bar{S}_{i}\right)-y_{p d c}\right)^{2}},
$$

with $\bar{S}_{i}=\max _{j=1, \ldots, N_{s}}\left(S_{i j}\right), i=1, \ldots, N_{p}$. The constraints (33) are introduced to ensure that each PMU measures one and only one partition of the state vector. The constraints (34) are introduced to avoid that the same partition is measured by more than one PMU.

Remark. The optimization problem may be solved by using any available mixed integer programming methods (such as Branch and Bound methods, simulated annealing, genetic algorithms ...), (see Gou (2008) for example).

\section{DYNAMICAL STATE-OBSERVER DESIGN}

The here-proposed derivation of a centralized state observer for wide-area monitoring is based on both the nonlinear differential model (18)-(20) and the use of an Extended Kalman Filter (see Gibbs (2011) for example):

$$
\begin{aligned}
& \dot{\hat{x}}=F(\hat{x}, \hat{z}, u)+L_{1}(\hat{x}, \hat{z}, u, w)(y-H(\hat{x}, \hat{z})), \\
& \dot{\hat{z}}=-\frac{\partial G^{-1}}{\partial z}\left[\frac{\partial G}{\partial x} F(\hat{x}, \hat{z}, u)+\frac{\partial G}{\partial w} \hat{\dot{w}}\right.
\end{aligned}
$$




$$
\begin{aligned}
& \left.+\frac{1}{\epsilon} G(\hat{x}, \hat{z}, w)\right]+L_{2}(\hat{x}, \hat{z}, u, w)(y-H(\hat{x}, \hat{z})), \\
\hat{\dot{w}} & =k_{c}(w-\hat{w}),
\end{aligned}
$$

where $L_{1}$ and $L_{2}$ gain matrices are obtained from the solution $P(t)$ of the following differential Riccati equation:

$$
\begin{aligned}
\dot{P}(t) & =P(t) A_{a}(\hat{x}, \hat{z}, u, w)^{T}+A_{a}(\hat{x}, \hat{z}, u, w) P(t) \\
& -P(t) C_{a}(\hat{x}, \hat{z})^{T} R^{-1} C_{a}(\hat{x}, \hat{z}) P(t)+Q, \\
P(0) & =Q_{0},
\end{aligned}
$$

where $A_{a}$ and $C_{a}$ are the Jacobian matrices of the system vector field (18)-(19) and the output vector $H(x, z)$ with respect to the state vector $\left(x^{T}, z^{T}\right)^{T}$, evaluated around the estimated trajectory $\hat{x}, \hat{z}, u, w$. The matrices $Q_{0}, Q$ and $R$ are chosen in order to represent the covariance matrix of the initial state, of the state equation disturbance and of the measurement disturbance, respectively.

The gain matrix $L=\left(\begin{array}{c}L_{1} \\ L_{2}\end{array}\right)$ is given by

$$
L(\hat{x}, \hat{z}, u, w)=P(t) C_{a}^{T}(\hat{x}, \hat{z}) R^{-1} .
$$

(41) is the nonideal differentiator which provides an estimate of $\dot{w}$.

\section{Some remarks.}

(1) There is no need to invert the algebraic "flow" equations (12) of the grid (for example by using a continuation method) since the observer is fully differential.

(2) Another advantage induced by (14) is the fact that if the generator state $x$ is stable and observable in the linear sense (for linearized dynamics) ${ }^{4}$, the grid state $z$ will always be at least detectable, what ensures local convergence of the observer.

(3) Furthermore, it can also be easily shown that under the assumption that the EKF state observer converges, the initial state estimate is not supposed to satisfy (12) to get an estimate of $x, z$ such that $(\hat{x}(t), \hat{z}(t)) \rightarrow(x(t), z(t))$ of the algebraic -differential model (11)-(12), when $t \rightarrow+\infty$.

\section{APPLICATION TO A 5-BUS POWER SYSTEM}

We consider now the 5-bus power system proposed in Bergen (2000), p. 357 (see Fig. 2). Without restriction and for simplification purpose, we assume that the series line impedances are $z_{L}=r_{L}+j x_{L}=0.0099+j 0.099$ and we neglect the capacitive (shunt) impedances. Bus 1 is the slack bus. Table 1 shows the generator and exciter data. Table 2 shows the other bus data. We assume that

Table 1. Generator and exciter data.

\begin{tabular}{ccccccccc}
\hline Generator & $H$ & $D$ & $T_{d 0}^{\prime}$ & $x_{d}$ & $x_{q}$ & $x_{d}^{\prime}$ & $K_{a}$ & $T_{a}$ \\
\hline 2 & 5.0 & 1.0 & 5.0 & 1.5 & 1.2 & 0.4 & 20 & 0.05 \\
\hline 3 & 5.0 & 0.0 & 6.0 & 1.4 & 1.35 & 0.3 & 20 & 0.05 \\
\hline
\end{tabular}

the system is equipped with some traditional $(P, Q)$ sensors, which measure both the active and reactive powers transmitted between bus 2 and bus 3 , bus 2 and bus 4 ,

4 Observability of $x$ is obtained by measuring at least the relative speeds and the exciter voltages of the generators.

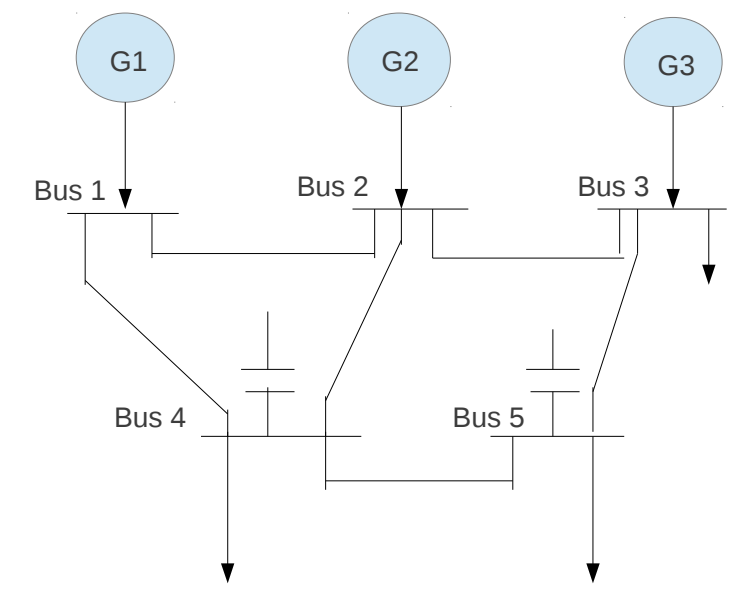

Fig. 2. A 5-bus power system with 3 generators.

Table 2. Bus data.

\begin{tabular}{ccccc}
\hline & & & & \\
Bus & Specified Voltage & Load & Shunt & Generation \\
\hline 1 & 1.0 & & & \\
\hline 2 & 1.0 & $0.2+\mathrm{j} 0.1$ & & 0.8830 \\
\hline 3 & 1.0 & $1.7137+\mathrm{j} 0.5983$ & $\mathrm{j} 1.0$ & \\
\hline 4 & & $1.7355+\mathrm{j} 0.5496$ & $\mathrm{j} 1.0$ & \\
\hline 5 & &
\end{tabular}

bus 3 and bus 5, respectively. In addition, the exciter voltages of generators $G_{2}$ and $G_{3}$ are measured, together with the relative speeds of the generators. The objective is to compute the best location of 2 PMUs dedicated to voltage phasor measurement at two of the buses, together with the optimal location of a PDC connected to the PMUs. The communication infrastructure cost is assumed to be an affine function of the distances between each of the 2 PMUs and the PDC. Table 3 shows the bus communication costs per $\mathrm{km}$, which correspond to the costs per $\mathrm{km}$ of the links between the PMUs connected to the buses, and the geographical coordinates of each bus in a 0xy frame. The communication cost differs from a given bus to another due to some civil engineering constraints for example. We con-

Table 3. Bus communication infrastructure cost per $\mathrm{km}$ and geographical data.

\begin{tabular}{ccc} 
Bus & Cost & Coordinates xy $($ in $\mathrm{km})$ \\
\hline 2 & 1 & 70,100 \\
\hline 3 & 1.1 & 90,190 \\
\hline 4 & 1.2 & 20,80 \\
\hline 5 & 1.5 & 35,170 \\
\hline
\end{tabular}

sider 10 scenarii obtained around the nominal operating point by randomly changing the load and the generation set points using a uniform distribution. Since the number of configurations is limited, the optimization problem can be solved by enumeration. Table 4 shows the obtained results. While (bus 3, bus 5) is the best PMU location from the observability point of view, the best trade-off observability / communication infrastructure cost is location (bus 2, bus 4). The EKF observer has also been tested with the optimal configuration of the sensors and $\epsilon=0.002$, when the power system reacts to a sudden load 
Table 4. Optimization cost values w.r.t. PDC and PMU location, with $\bar{d}=30 \mathrm{~km}$

\begin{tabular}{ccccc}
$\begin{array}{c}\text { Location } \\
(\text { bus } 1, \text { bus2 })\end{array}$ & $\begin{array}{c}E\left[\text { trace }\left(W_{o}\right)\right] \\
\text { with } \epsilon=0.002\end{array}$ & $C_{c}$ & $\begin{array}{c}\text { Total } \\
\text { cost }\end{array}$ & $\begin{array}{c}\text { PDC } \\
\text { location }\end{array}$ \\
\hline$(2,3)$ & 17332.26 & 98.41 & 17233.85 & $76.51,129.28$ \\
\hline $\mathbf{2 , 4 )}$ & 17325.45 & 58.62 & $\mathbf{1 7 2 6 6 . 8 3}$ & $42.15,88.86$ \\
\hline$(2,5)$ & 17328.31 & 102.39 & 17225.92 & $56.58,126.83$ \\
\hline$(3,4)$ & 17332.13 & 153.46 & 17178.67 & $73.89,164.69$ \\
\hline$(3,5)$ & $\mathbf{1 7 3 3 4 . 9 8}$ & 75.78 & 17259.20 & $61.80,179.75$ \\
\hline$(4,5)$ & 17328.17 & 127.86 & 17200.31 & $24.93,109.59$ \\
\hline
\end{tabular}

drop of $0.8+$ j0.8 p.u at each buses, without considering frequency control mechanisms. Fig. 3 and 4 show how well the observer behaves in response to this disturbance.

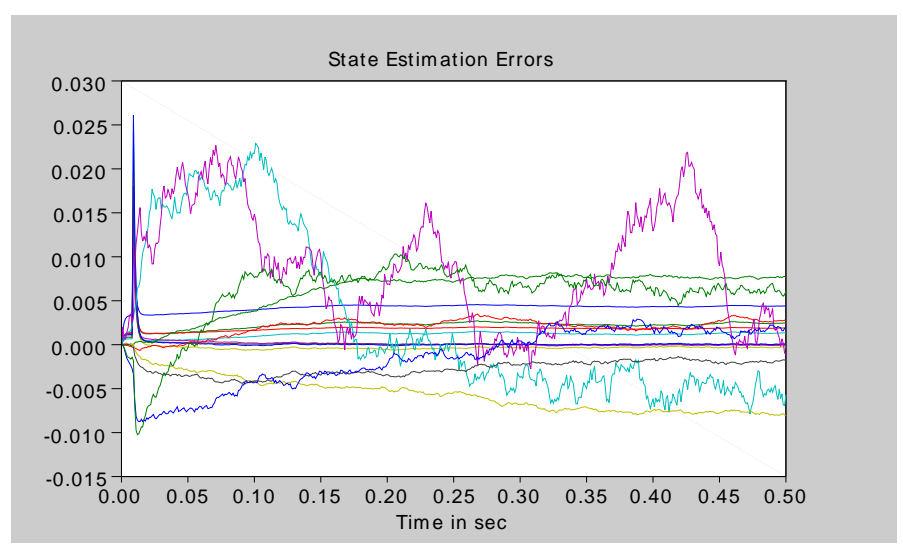

Fig. 3. EKF estimation of the generator angles and relative speeds.

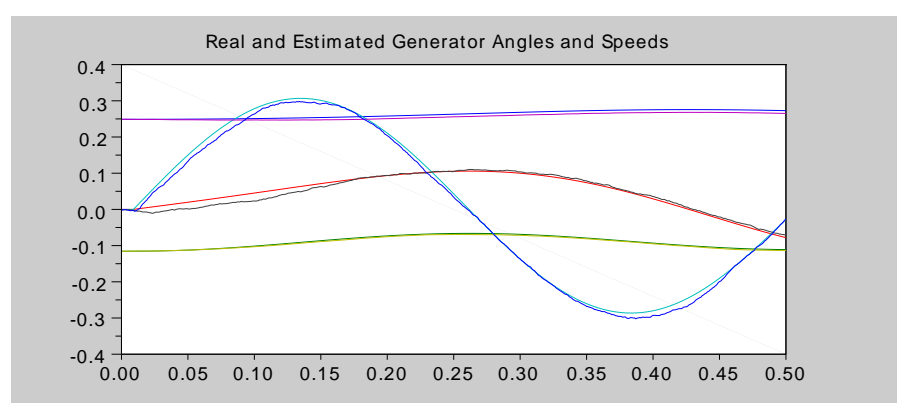

Fig. 4. EKF estimation of the bus voltages.

\section{CONCLUSIONS AND PERSPECTIVES}

In this paper, a methodology for design an optimal monitoring architecture using a limited number of PMUs has been proposed. The optimal design problem consists in maximizing the observability index of the power system (which is based on the trace of the linear observability gramian), while minimizing the communication infrastructure cost. Furthermore, a nonlinear dynamical EKF stateobserver is proposed, which does not require any on-line "nonlinear inversion" technique. Further researches will be devoted to the design of a distributed version of the hereproposed EKF state observer suitable for both reducing the computational cost and taking advantage of the distributed nature of wide-area power systems.

\section{REFERENCES}

Akyol, B.A., Kirkham, H., Clements, S.L., and Hadley, M.D. (2010). A Survey of Wireless Communications for the Electric Power System. Report for the U.S. Department of Energy, 2009.

Baldwin, T., Mili, L., Boisen, J., and Adapa, R. (1993). Power system observability with minimal phasor measurement placement. IEEE Trans. on Power Systems, vol. 8, no. 2, pp. 707-715, 1993.

Bergen, A.R., and Vittal, V. (2000). Power system analysis. Second edition. Prentice Hall, 2000.

Georges, D. (1995). The use of observability and controllability gramians or functions for optimal sensor and actuator location in finite-dimensional systems. Proceedings of the 34th IEEE Conference on Decision and Control, 1995 .

Georges, D. (2011). Energy Minimization and Observability Maximization in Multi-Hop Wireless Sensor Networks. Proceedings of the 18th IFAC World Congress, Milano, Italy, 2011.

Gibbs, B. (2011). Advanced Kalman filtering, Leastsquares and modeling. J. Wiley and Sons, Inc. 2011.

Gou, B. (2008). Generalized integer linear programming formulation for optimal pmu placement. IEEE Trans. on Power Systems, vol. 23, no. 3, pp. 1099-1104, 2008.

Huang, Z., Schneider, K., and Nieplocha, J. (2007). Feasibility studies of applying Kalman filter techniques to power system dynamic state estimation. Proceedings of the International Power Engineering Conference 2007, 2007.

Ilic, M., and Zaborszky, J. (2000). Dynamics and control of large electric power systems. John Wiley and Sons, Inc., 2000.

Narendra, K., and Weekes, T. (2008). Phasor Measurement Unit (PMU) Communication Experience in a Utility Environment. Conference on Power Systems, Winnipeg, October 19-21, Cigre, 2008.

Nguyen, D.T., Georges, D., and Tran, Q.T. (2008). An Energy Approach to Optimal Selection of Controllers and Sensors in Power System. International Journal of Emerging Electric Power Systems, vol. 9, 2008, [hal-00370850], http://www.bepress.com/ijeeps/vol9/iss6/art2.

Phadke, A., Thorp, J., and Adamiak, M. (1983). A new measurement technique for tracking voltage phasors, local system frequency, and rate of change of frequency. IEEE Trans. on Power Apparatus and Systems, vol. 102, no. 5, pp. 1025-1038, may 1983.

$\mathrm{Xu}$, B., and Abur, A. (2004). Observability analysis and measurement placement for systems with pmus. IEEE PES, 10-13 2004, pp. 943-946, vol.2.

Zhou, J., Salomon, G., and Wu, E. (1999). Balanced realization and model reduction for unstable systems. Int. J. Robust Nonlinear Control, Vol. 9, no. 3, pp. 183198, 1999. 